\title{
DIAGNÓSTICOS E PROPOSTAS DE INTERVENÇÕES DE ENFERMAGEM AOS PACIENTES EM PÓS OPERATÓRIO IMEDIATO DE CIRURGIA ELETIVA*
}

Kátia Cilene Godinho Bertoncello', Bruna Sávioº ${ }^{2}$ Juliana Martins Ferreira ${ }^{3}$

Lúcia Nazareth Amante ${ }^{4}$, Eliane Regina Pereira do Nascimento ${ }^{1}$

${ }^{1}$ Enfermeira. Doutora em Enfermagem. Universidade Federal de Santa Catarina. Florianópolis-SC-Brasil.

${ }^{2}$ Enfermeira. Residente do Hospital de Clinicas da Universidade Federal do Paraná. Curitiba-PR-Brasil.

${ }^{3}$ Enfermeira. Clínica Dircksen. Florianópolis-SC-Brasil.

RESUMO: Objetivou-se identificar os diagnósticos de enfermagem apresentados por pacientes em pós-operatório imediato de cirurgia eletiva e propor intervenções de enfermagem. Realizou-se uma pesquisa quantitativa, exploratória e descritiva. Evoluídos 27 pacientes internados na clínica cirúrgica de um hospital universitário no Sul do Brasil, maiores de 18 anos, ambos os sexos, que estivessem em pós-operatório imediato no período de março e abril de 2011; e que não apresentasse complicações, nem re-operações. Utilizando o raciocínio de diagnóstico de Risner, identificaram-se sete diagnósticos segundo a North American Nursing Diagnosis Association. Propôs-se 62 intervenções de enfermagem baseadas na Nursing Interventions Classification, que pudessem propiciar redução de danos a esses pacientes específicos. As intervenções, notadas pelos diagnósticos proporcionam autonomia ao enfermeiro e um cuidado individualizado, fundamentados nos fatores relacionados e nas características definidoras identificadas.

DESCRITORES: Diagnóstico de enfermagem; Cirurgia geral; Cuidados pós-operatórios.

\section{NURSING DIAGNOSES AND PROPOSAIS FOR NURSING INTERVENTIONS FOR PATIENTS INTHE IMMEDIATE POST- OPERATIVE PERIOD FOLOWING EFCTIVE SURGERY}

ABSTRACT: This study aimed to identify the nursing diagnoses presented by patients in the immediate postoperative period following elective surgery, and to propose nursing interventions. It is a quantitative, exploratory and descriptive study. It involved 27 patients receiving inpatient treatment in the surgical department of a university hospital in the south of Brazil, of both sexes, who were in the immediate postoperative period, in March - April 2011; and who did not present complications or need to be re-operated on. Using Risner's model of diagnostic reasoning, seven North American Nursing Diagnosis Association diagnoses were identified. A total of 62 nursing interventions, based in the Nursing Interventions Classification, which could lead to a reduction in harm to these specific patients, were proposed. The interventions, grounded in the diagnoses, allow the nurse to work with autonomy and to provide individualized care, based in the factors reported and in the defining characteristics identified.

DESCRIPTORS: Nursing diagnoses; General surgery; Postoperative care.

\section{DIAGNÓSTICOSY PROPUESTAS DE INTERVENCIONES DE ENFERMERÍA A LOS PACIENTES EN POSOPERATORIO INMEDIATO DE CIRUGÍA ELECTIVA}

RESUMEN: Estudio cuyo objetivo fue identificar los diagnósticos de enfermería presentados por pacientes en posoperatório inmediato de cirugía electiva y proponer intervenciones de enfermería. Se realizó una investigación cuantitativa, exploratoria y descriptiva. Participaron 27 pacientes internados en la clínica cirúgica de un hospital universitario en el Sur de Brasil, mayores de 18 años, ambos los sexos, que estuvieran en posoperatório inmediato en el periodo de marzo y abril de 2011; y que no presentasen complicaciones o re-operaciones. Utilizando el raciocinio de diagnóstico de Risner, se identificaron siete diagnósticos de acuerdo a North American Nursing Diagnosis Association. Fueron propuestas 62 intervenciones de enfermería basadas en la Nursing Interventions Classification, que pudiesen propiciar una reducción de daños a esos pacientes específicos. Las intervenciones, fundamentadas por los diagnósticos, posibilitan autonomía al enfermero y un cuidado individualizado, fundamentado en factores relacionados y en las características definidoras identificadas. DESCRIPTORES: Diagnóstico de enfermería; Cirugía general; Cuidados posoperatorios

*Artigo originado do Trabalho de Conclusão Curso do Curso de Graduação em Enfermagem da

Universidade Federal de Santa Catarina, 2012.

Autor Correspondente:

Kátia Cilene Godinho Bertoncello

Universidade Federal de Santa Catarina

Rua Pedro Vieira Vidal, 280 - 88040-010 Florianópolis-SC-Brasil

E-mail: kbertoncello@yahoo.com.br
Recebido: 04/10/2013

Finalizado: 26/06/2014 


\section{INTRODUÇÃO}

A cirurgia, para qualquer pessoa, é uma situação estressante e complexa, e sua história, vem de muito tempo ${ }^{(1)}$. Os procedimentos cirúrgicos evoluem a cada dia, e se tornaram mais seguros, com a maior exatidão dos diagnósticos pré-operatórios; segurança na indicação cirúrgica, como terapia e planejamento da técnica cirúrgica. Isso contribui para um pós-operatório mais rápido para os pacientes ${ }^{(2)}$.

Dessa forma, os indivíduos, que permanecem em internação hospitalar no pós-operatório, requerem maiores cuidados, mesmo os pacientes de cirurgia eletiva, visto que a internação é necessária, geralmente, para os submetidos a cirurgias de médio a grande porte e que apresentam distúrbios clínicos concomitantes ${ }^{(1)}$.

A enfermagem perioperatória é uma especialidade que abrange vários aspectos do cuidado em enfermagem. A atuação do enfermeiro, nesse período, é efetuar uma avaliação em cada uma das suas fases, o pré-operatório, o intra-operatório e o pós-operatório( ${ }^{(3)}$.

A expressão diagnóstico de enfermagem (DE), tendo por definição: "julgamento clínico que proporciona a base para seleção das intervenções de enfermagem, visando alcançar resultados"(4:103), passou a ser utilizada formalmente na década de 1950, no contexto de diagnósticos clínicos. Em 1973 foram identificadas necessidades dos pacientes no âmbito da enfermagem e estabeleceuse termos descritivos únicos, chamados de diagnósticos de enfermagem, posteriormente com a criação a North American Nursing Diagnosis Association (NANDA Internacional) ${ }^{(5)}$.

Em concordância, a Nursing Interventions Classification (NIC)(6), a Nursing Outcomes Classification (NOC), a NANDA Internacional (NANDA I), tem como objetivo padronizar a linguagem e a comunicação entre enfermeiro e equipe de enfermagem. Além de otimizar o tempo na elaboração dos diagnósticos de enfermagem, permite que o enfermeiro passe mais tempo nas atividades assistenciais ${ }^{(7)}$.

O conhecimento teórico e científico, juntamente com a NIC e a NANDA I, tornamse instrumento que possibilita ao profissional maior viabilidade de aplicar a assistência de enfermagem de forma qualificada e integral ${ }^{(8)}$.
O Conselho Federal de Enfermagem, pela Resolução n. 358/2009, também se manifestou, frente a esta problemática, contribuindo com o atendimento de enfermagem, quando determinou a obrigatoriedade da aplicação da Sistematização da Assistência de Enfermagem (SAE) para todos os pacientes. Todavia, esclarece que essa é uma atividade privativa do enfermeiro, porque é um método utilizado para identificação do processo de saúde/doença do usuário dos serviços de saúde, contribuindo, assim, para a promoção, prevenção, recuperação e reabilitação do paciente, de acordo com os princípios do Sistema Único de Saúde ${ }^{(9)}$.

Entende-se que os diagnósticos de enfermagem e as possíveis intervenções, relacionados com indivíduos em pós-operatório imediato, é uma temática ainda pouco abordada na prática profissional, assim como os demais assuntos que abrangem a SAE.

Frente a esta problemática, justificou-se o desenvolvimento dessa investigação que teve como objetivo identificar os diagnósticos de enfermagem apresentados por pacientes em pós operatório imediato de cirurgia eletiva, utilizando a Taxonomia II da NANDA I ${ }^{(4)}$ e propor intervenções de enfermagem para o cuidado destes pacientes, de acordo com a $\mathrm{NIC}^{(6)}$.

\section{MÉTODO}

Trata-se de uma pesquisa quantitativa, exploratória e descritiva, desenvolvida na unidade da clínica cirúrgica II, de um hospital universitário, totalmente público, localizado no município de Florianópolis-SC-Brasil.

A amostra constituiu-se de 27 pacientes internados na clínica cirúrgica II, que estavam em pós-operatório imediato de cirurgia eletiva, durante o período de coleta de dados que ocorreu entre os meses de março e abril de 2011. Os critérios de inclusão foram: ser paciente maior de 18 anos, que estivessem em pós-operatório imediato de cirurgia eletiva no período de coleta de dados, que não se encontrasse gestante, e que não tivessem complicações no pós-operatório ou re-operação.

A investigação foi desenvolvida em três etapas. A primeira constitui-se da coleta de dados, por meio de consulta ao prontuário (identificando idade, sexo, tipo, dia e hora da cirurgia eletiva 
realizada), ao histórico de enfermagem (buscando complicações pós-operatórias, re-operações, gestação) e por fim, foi realizada a evolução de enfermagem em instrumento eletrônico próprio da unidade cirúrgica dos 27 pacientes que atenderam os critérios de inclusão.

A segunda etapa consistiu na identificação do diagnóstico de enfermagem, utilizando-se o processo de raciocínio diagnóstico de $\operatorname{Risner}^{(10)}$, cujas etapas são a análise e a síntese.

A etapa da análise é aquela na qual o material coletado é separado em partes e examinado criticamente. Nessa fase, existem duas etapas: categorização dos dados, que são organizados de maneira lógica, sistematizada e podem ser embasados em diferentes modelos conceituais; identificação de lacunas de dados, compreendida como sendo a etapa na qual os dados incompletos são avaliados, podendo haver necessidade de nova coleta de dados, o que, neste estudo, não foi necessário. Cabe ressaltar que nesta pesquisa a fase de análise foi realizada durante a coleta de dados, através do instrumento de registro de evolução de enfermagem.

A etapa da síntese é a fase na qual o processo de raciocínio diagnóstico de enfermagem é realizado, considerando as seguintes etapas: agrupamento de dados relevantes e comparação dos dados agrupados com teorias, modelos e conceitos, ambos consistem na maior etapa da fase de síntese, em que os dados coletados sobre o paciente são interpretados e comparados com normas e padrões. Identificação de desvios ou potencialidades de saúde (inferência ou hipótese) é a etapa do julgamento clínico, é a elaboração de hipóteses diagnósticas, considerando as conclusões esboçadas sobre os problemas do paciente após comparação com teorias, modelos e conceitos; proposição de relações etiológicas. Nessa etapa, os fatores que influenciaram ou contribuíram para a elaboração das hipóteses (inferências) são identificados ${ }^{(10)}$.

Nesta investigação, as inferências ocorreram após a coleta de dados, quando foram relacionados os problemas apresentados dentro dos fatores de risco individuais de cada paciente, em pósoperatório imediato de cirurgia eletiva.

Após a aplicação do processo de raciocínio diagnóstico, nos dados coletados, construiuse as afirmativas diagnósticas, utilizando a Taxonomia II da Classificação NANDA I(4) com suas características definidoras, fatores relacionados ou fatores de risco.

$\mathrm{Na}$ terceira e última etapa, diante das identificações diagnósticas, encontradas, especificamente para esses indivíduos em pósoperatório imediato, buscou-se a elaboração de uma proposta de intervenções de enfermagem, segundo a Classificação da $\mathrm{NIC}^{(6)}$, para os DE que obtiveram a frequência igual e superior a $50 \%$ nos 27 pacientes investigados.

Vários fatores são considerados imprescindíveis para a seleção de intervenção de enfermagem de acordo com a $\mathrm{NIC}^{(6)}$, adotou-se para tomada de decisão, neste estudo, os seis principais: os resultados almejados, as características dos DE, a pesquisa básica para a intervenção, a viabilidade da ação, a adesão do paciente a terapêutica proposta e a capacidade do enfermeiro.

A pesquisa foi aprovada pelo Comitê de Ética em Pesquisa com Seres Humanos da PróReitoria de Pesquisa e Extensão da Universidade Federal de Santa Catarina, sob protocolo $\mathrm{n}^{\circ}$ 1169/2010. Os participantes foram convidados a participar, todos aceitaram, e assinaram o Termo de Consentimento Livre e Esclarecido, a todo o momento foi garantindo-lhes o direito de desistência, assim como o completo anonimato.

\section{RESULTADOS}

Dos 27 pacientes, que participaram da investigação, 15(56\%) eram do sexo masculino e $12(44 \%)$ do sexo feminino. Em relação à idade, quatro $(14,8 \%)$ pacientes eram menores de 30 anos, $15(55,5 \%)$ estavam entre 30 e 60 anos e os oito (29,6\%) estavam acima de 60 anos.

Os pacientes em pós-operatório eram provenientes de especialidades médicas distintas. Os internados pela cirurgia plástica constituíram a maior parte da amostra, 10(37\%) dos pacientes, seguidos pela cirurgia urológica sete(25,9\%), cirurgia proctológica cinco $(18,5 \%)$ e cirurgia vascular quatro $(14,8 \%)$.

Foram identificados nesses pacientes 18 diagnósticos de enfermagem, no período pósoperatório imediato, sendo 12 diagnósticos reais de enfermagemeseis diagnósticos deriscodeenfermagem.

Frente ao corte escolhido para esta investigação, de igual e superior a 50\% dos resultados encontrados, nos 27 pacientes investigados, apresentou-se, portanto, sete (51,8\%) diagnósticos reais de enfermagem, seguido de seus fatores 
de risco e suas características definidoras manifestadas pelos indivíduos, formulando os enunciados diagnósticos ${ }^{(4)}$.

O DE déficit no autocuidado para banho teve relação com a dor em 19(70,3\%) dos pacientes, ao prejuízo músculo esquelético em cinco (18,5\%) e a fraqueza em um (2,7\%), esses manifestados por incapacidade de acessar o banheiro, de lavar o corpo, de obter a fonte da água, de pegar artigos para o banho, de regular a água do banho e de secar o corpo.

Tabela 1 - Distribuição dos pacientes em pós-operatório imediato, submetidos à cirurgia eletiva, segundo os enunciados dos diagnósticos reais. Florianópolis-SC-Brasil, 2011

\begin{tabular}{lcc}
\hline Enunciado do Diagnóstico Real & $\mathbf{n}$ & $\mathbf{\%}$ \\
\hline $\begin{array}{l}\text { 1. Dor aguda relacionada a agentes lesivos físicos, manifestada por } \\
\text { evidência observada de dor, mudanças de apetite, relato verbal de dor }\end{array}$ & 27 & 100 \\
\hline $\begin{array}{l}\text { 2. Integridade da pele prejudicada relacionada a fatores mecânicos, } \\
\text { manifestada por rompimento as superfície da pele, invasão das } \\
\text { estruturas do corpo }\end{array}$ & 27 & 100 \\
\hline $\begin{array}{l}\text { 3. Mobilidade física prejudicada relacionada à dor manifestada } \\
\text { por amplitude limitada de movimento, dificuldade para virar-se, } \\
\text { movimentos lentos }\end{array}$ & 23 & 75,1 \\
\hline $\begin{array}{l}\text { 4. Déficit no autocuidado para o banho relacionado à dor, manifestado } \\
\text { por amplitude limitada de movimento, dificuldade para virar-se, } \\
\text { movimentos lentos }\end{array}$ & 19 & 70,3 \\
\hline $\begin{array}{l}\text { 5. Déficit no autocuidado para higiene íntima relacionado à dor, } \\
\text { manifestada por incapacidade de acessar o banheiro, de lavar o corpo, } \\
\text { de obter a fonte de água, de pegar artigos para o banho, de regular a } \\
\text { água do banho, de secar o corpo }\end{array}$ & 19 & 70,3 \\
\hline $\begin{array}{l}\text { 6. Déficit no autocuidado para vestir-se relacionado à dor, manifestada } \\
\text { por capacidade prejudicada de colocar itens de vestuário necessário, } \\
\text { capacidade prejudicada de tirar itens de vestuário necessário }\end{array}$ & 18 \\
\hline $\begin{array}{l}\text { 7. Constipação relacionada a mudanças recentes de ambiente, } \\
\text { manifestada por incapacidade de eliminar fezes, náusea, vômito, dor } \\
\text { abdominal, indigestão, mudanças no padrão intestinal }\end{array}$ & 14 & 66,6 \\
\hline
\end{tabular}

\section{DISCUSSÃO}

Nesta pesquisa o diagnóstico de enfermagem de Dor aguda esteve relacionado a agentes lesivos e manifestado por evidência observada de dor, mudanças de apetite e relato verbal de dor ${ }^{(4)}$. Logo, estas manifestações clínicas são justificadas por estarem em pós-operatório imediato, cuja presença do trauma é provocada pela lesão tecidual cirúrgica muito recente.

Quando esse diagnóstico é identificado pelo enfermeiro, vários fatores devem ser observados, tais como: idade, sexo, nível de instrução, ocupação e raça ${ }^{(11)}$ já que a dor é uma das sensações mais íntimas e exclusivas experimentadas pelo ser humano, pois envolve vários componentes sensoriais, afetivos e cognitivos, sociais e comportamentais e será sempre subjetiva ${ }^{(12)}$.

Deste modo, a avaliação da experiência dolorosa é bastante difícil. Segundo a Associação
Internacional para Estudos da Dor, a dor é

uma experiência sensorial e emocional desagradável, associada a um dano real ou potencial dos tecidos, ou descrita em termos de tais lesões ${ }^{(12: 105)}$.

Cada indivíduo aprende a utilizar esses termos através de suas experiências prévias traumáticas, assim a dor nunca pode ser subjugada, quando não tratada adequadamente afeta a qualidade de vida dos doentes e consequentemente toda sua recuperação no pós-operatório(12).

Como se pode observar, por meio do enunciado dos diagnósticos de enfermagem, a dor dos pacientes em pós-operatório imediato tinha relação com: mobilidade física prejudicada e déficit no autocuidado, seja para banho, vestir-se ou higiene íntima.

Quando falamos do diagnóstico de enfermagem de déficit no autocuidado, nessa pesquisa, ele foi identificado em três situações: 
déficit no autocuidado para banho, déficit no autocuidado para higiene íntima e déficit no autocuidado para vestir-se.

O DE déficit no autocuidado para higiene íntima, manifestado por incapacidade de chegar ao vaso sanitário ou à cadeira higiênica; de dar descarga no vaso sanitário; de fazer uma higiene íntima apropriada; de levantar-se do vaso sanitário ou da cadeira higiênica; de manipular as roupas para realizar a higiene íntima; e incapacidade de sentar-se no vaso sanitário ou na cadeira higiênica, também teve relação com os mesmos aspectos, praticamente na mesma proporção alterando apenas no que faz relação com fraqueza, a qual se apresentou em dois pacientes.

Já o DE déficit no autocuidado para vestir-se, que é manifestado por capacidade prejudicada de colocar itens de vestuário; capacidade prejudicada de tirar itens de vestuário foi relacionada à dor em $18(66,6 \%)$ dos casos e a fraqueza em um paciente.

Outro DE, identificado em todos os pacientes, foi Integridade da pele prejudicada, relacionado a fatores mecânicos e manifestado por rompimento das superfícies da pele e invasão das estruturas corpóreas $^{(4)}$.

Segundo a definição de NANDA I ${ }^{(4)}$ a integridade da pele prejudicada tem por definição epiderme e/ou derme alterada. Logo, é um estado em que o indivíduo em pós-operatório imediato apresenta $^{(13)}$.

O princípio do cuidado de enfermagem é justificado, pois os tecidos são grupos de células especializadas que se unem para desempenhar funções específicas. As interações complexas entre a derme e a epiderme levam a mensagem de uma para a outra no caso de lesão que necessite de correção ${ }^{(14)}$. A intervenção de enfermagem nesse aspecto, em pacientes em pós-operatório imediato, é fundamental a fim de evitar novas lesões em um tecido prejudicado, como as úlceras de pressão, além de promover cuidados ou reparação do tecido.

Ainda, entre os diagnósticos de enfermagem que tiveram $51,8 \%(14)$ de frequência foi identificado o DE constipação, relacionado às mudanças recentes de ambiente, manifestado por incapacidade de eliminar fezes, acompanhados ou não de náusea, vômito, dor abdominal e indigestão.

Essas mudanças não estão apenas relacionadas à mudança de ambiente em si, mas sim mudança completa na alimentação e hábitos do paciente. Por isso, constipação é uma condição muito frequente em pacientes hospitalizados, já que existe uma clara diferença na dieta dos pacientes em ambiente hospitalar e também porque a mobilidade física do paciente fica diminuída, fatores que interferem, diretamente, na função intestinal $^{(15)}$.

A NIC ${ }^{(6)}$ aborda a assistência direta ou indireta, focalizando o indivíduo, família e a comunidade, ela é o arranjo das atividades de enfermagem, de acordo com os seus grupos ou conjuntos, levando em consideração as relações e determinações que designam as intervenções de um grupo. A seleção de uma intervenção requer tomada de decisão direta do enfermeiro para um paciente em particular.

Portanto, frente a esta tomada de decisão, conforme as características físicas e estruturais da unidade cirúrgica, as características definidoras apresentadas pelos pacientes em pós-operatório imediato de cirurgia eletiva e fundamentos das Intervenções da $\mathrm{NIC}^{(6)}$ apresentou-se uma proposta de ações, para cada um dos sete DE identificados. 


\begin{tabular}{|c|c|}
\hline Diagnósticos de Enfermagem ${ }^{(4)}$ & Intervenções de Enfermagem ${ }^{(6)}$ \\
\hline 1.Dor aguda & $\begin{array}{l}\text { Realizaruma avaliaçãocompleta da dor, incluindolocal, características, início/duração, } \\
\text { frequência, qualidade, intensidade e gravidade, além de fatores precipitadores; } \\
\text { Observar a ocorrência de indicadores não verbais de desconforto, em especial } \\
\text { nos pacientes incapazes de se comunicar com eficiência; } \\
\text { Usar estratégias terapêuticas de comunicação para reconhecer a experiência } \\
\text { de dor e transmitir aceitação da resposta do paciente a dor; } \\
\text { Investigar os fatores que aliviam/pioram a dor; } \\
\text { Informar sobre a dor, suas causas, duração e desconfortos antecipados em } \\
\text { decorrência dos procedimentos; } \\
\text { Controlar fatores ambientais capazes de influenciar a resposta do paciente ao } \\
\text { desconforto; } \\
\text { Reduzir ou eliminar fatores que precipitam ou aumentam a experiência de dor; } \\
\text { Encorajar o paciente a monitorar a própria dor e intervir de forma adequada; } \\
\text { Administrar analgésicos, quando prescritos; } \\
\text { Aplicar calor/frio quando apropriado. }\end{array}$ \\
\hline 2.Mobilidade física prejudicada & $\begin{array}{l}\text { Determinar a capacidade atual do paciente em transferir-se; } \\
\text { Selecionar a técnica de transferência adequada ao paciente; } \\
\text { Orientar o paciente sobre todas as técnicas apropriadas, almejando atingir o } \\
\text { maior nível de independência; } \\
\text { Orientar o indivíduo quanto ao uso auxiliares da deambulação; } \\
\text { Identificar os métodos de prevenção de lesão durante as transferências; } \\
\text { Usar a mecânica corporal correta durante os movimentos; } \\
\text { Manter o corpo do paciente no alinhamento correto durante os movimentos; } \\
\text { Auxiliar o paciente a deambular usando o corpo como muleta humana, } \\
\text { conforme apropriado; } \\
\text { Avaliar o paciente ao término da transferência quanto ao alinhamento } \\
\text { corporal correto, a não oclusão de sondas e drenos, roupas de cama com } \\
\text { rugas e dobras, pele desnecessariamente exposta, nível de conforto adequado } \\
\text { para o paciente, laterais da cama levantadas e campainha ao alcance da mão. }\end{array}$ \\
\hline $\begin{array}{l}\text { 3.Déficit no autocuidado para } \\
\text { banho }\end{array}$ & $\begin{array}{l}\text { Dar assistência no banho; } \\
\text { Manter cuidados com as unhas, períneo, cabelos, olhos, ouvidos e pés; } \\
\text { Promover saúde oral; } \\
\text { Promover a mecânica corporal; } \\
\text { Melhorar a imagem corporal do paciente; } \\
\text { Melhorar a autoestima do paciente; } \\
\text { Auxiliar na utilização do vaso sanitário; } \\
\text { Auxiliar o paciente a vestir-se/arrumar-se; } \\
\text { Estimular o exercício de vestir-se. }\end{array}$ \\
\hline $\begin{array}{l}\text { 4.Déficit no autocuidado para } \\
\text { higiene íntima }\end{array}$ & $\begin{array}{l}\text { Dar assistência na higiene íntima; } \\
\text { Manter cuidados com períneo; } \\
\text { Promover a mecânica corporal; } \\
\text { Melhorar a imagem corporal do paciente; } \\
\text { Melhorar a autoestima do paciente; } \\
\text { Auxiliar na utilização do vaso sanitário. }\end{array}$ \\
\hline $\begin{array}{l}\text { 5.Déficit no autocuidado para } \\
\text { vestir-se }\end{array}$ & $\begin{array}{l}\text { Oferecer a roupa de modo que o paciente tenha acesso a elas (p.ex., junto ao leito); } \\
\text { Estar disponível para ajudar a vestir o paciente, se necessário; } \\
\text { Manter a privacidade enquanto o paciente se veste; } \\
\text { Reforçar as tentativas de vestir-se sozinho. }\end{array}$ \\
\hline 6.Integridade da pele prejudicada & $\begin{array}{l}\text { Realizar curativo com a medicação tópica, adequada; } \\
\text { Observar sinais e sintomas de infecção; } \\
\text { Observar e manter cuidados com áreas de pressão; } \\
\text { Hidratar a pele, quando necessário; } \\
\text { Fazer limpeza diária da incisão operatória; } \\
\text { Observar e registrar possíveis alterações nas extremidades inferiores; } \\
\text { Orientar ou posicionar o paciente para um melhor fluxo circulatório; } \\
\text { Observar sinais e sintomas de infecção em punção venosa; } \\
\text { Observar alterações na pele. }\end{array}$ \\
\hline
\end{tabular}


Monitorar os movimentos intestinais, inclusive a frequência, a consistência, a forma, o volume e a cor das fezes, se apropriado;

Monitorar ruídos hidroaéreos;

Registrar problemas intestinais preexistentes e o uso de laxantes;

Encorajar o aumento da ingestão de líquidos, a menos que seja contra indicado;

Avaliar o perfil dos medicamentos quanto a efeitos colaterais gastrointestinais;

Orientar o paciente/família para registrar cor, volume, frequência e consistência das fezes;

Orientar o paciente sobre os alimentos específicos que auxiliam na promoção da regularidade intestinais;

Orientar o paciente sobre alimentos ricos em fibras, se apropriado;

Oferecer líquidos quentes após as refeições, se apropriado;

Administrar enema ou irrigação, conforme prescrição médica;

Pesar o paciente regularmente;

Ensinar o paciente/família os processos digestivos normais;

Ensinar ao paciente/família o prazo para a solução da constipação.

\section{CONCLUSÃO}

Este estudo buscou, por meio de conhecimentos científicos, embasar uma forma de sistematização para o atendimento do paciente em pós-operatório imediato, de acordo com as características encontradas dentro do seu contexto de cirurgia eletiva.

Ao analisar essas características e identificar os diagnósticos reais de enfermagem, por meio do processo de raciocínio clínico, considerou-se esse método satisfatório, pois se obteve, para o total dos 27 pacientes, sete diagnósticos diferentes, em que $100 \%$ apresentaram: dor aguda e integridade da pele prejudicada; $85,1 \%(23)$ mobilidade física prejudicada; $70,3 \%(19)$ déficit no autocuidado para o banho e higiene íntima; 66,6\%(18) déficit no autocuidado para vestir-se e 51,8\%(14) constipação.

Para a tomada de decisão das intervenções de enfermagem, frente aos achados diagnósticos, seguiram-se os seis fatores recomendados pela NIC: os resultados almejados, as características dos diagnósticos, a pesquisa básica, a viabilidade da ação, a capacidade do enfermeiro e equipe de desenvolvê-las e os recursos materiais e humanos disponíveis no local da investigação.

Apresentaram-se para cada um dos sete diagnósticos reais presentes, em mais de 50\% dos pacientes em pós-operatório, as respectivas propostas de intervenções de enfermagem, buscando oferecer, como resultado deste estudo, um instrumento com um total de 62 ações para o enfermeiro assisti-los especificamente, individualizando o cuidado, a partir de seus fatores relacionados e de suas características definidoras identificadas.

Todavia, uma limitação dessa investigação foi o número baixo de pacientes estudados, justificada pela demanda de pacientes em pós-operatório imediato, durante o período de coleta de dados.

Contudo, acredita-se que os resultados deste estudo possam contribuir para a assistência de enfermagem prestada a estes indivíduos, em pós-operatório imediato de cirurgia eletiva, institucionalizados e atribuir maior cientificidade à prática e autonomia ao enfermeiro.

\section{REFERÊNCIAS}

1. Smeltzer SC, Bare BG. Bruner \& Studdarth. Tratado de Enfermagem Médico Cirúrgica. 12ª ed. Rio de Janeiro: Guanabara Koogan; 2012.

2. Torrati FG, Gois CFL, Dantas RAS. Estratégia no cuidado ao paciente cardíaco cirúrgico: avaliação do senso de coerência. Rev Esc Enferm USP. [Internet] 2010;44(3):739-44. [acesso em 17 jun 2011]. Disponível: http://dx.doi.org/10.1590/S008062342010000300027.

3. Calicchio LG. Práticas Recomendadas - SOBECC: Centro Cirúrgico, Recuperação Pós Anestésica, Centro de Materiais e Esterelização. $6^{a}$ ed. São Paulo: Sociedade Brasileira de Enfermeiros de Centro Cirúrgico, Recuperação Anestésica e Centro de Material de Esterilização (SOBECC); 2013. p. 232.

4. NANDA I. Diagnósticos de enfermagem da NANDA: definições e classificação - 2012 - 2014. 2011. Porto Alegre: Artmed; 2012. p. 458. 
5. Doenges ME, Moorhouse MF, Murr AC. Diagnósticos de Enfermagem. $12^{\mathrm{a}}$ ed. Rio de Janeiro: Guanabara Koogan; 2011. p. 727.

6. Bulechek MG, Butcher KH, Dochterman MJ. Classificação das Intervenções de Enfermagem (NIC). 5a ed. Mosby/Elsevier; 2011.

7. Sallum AMC, Santos JFL, Lima FD. Nursing diagnoses in trauma victims with fatal outcomes in the emergency scenario. Rev. Latino-Am. Enfermagem. [Internet]. 2012;20(1) [acesso em 20 dez 2013]. Disponível: http:// dx.doi.org/10.1590/S0104-11692012000100002

8. Bertoncello KCG, Cavalcanti CDK, Ilha P. Análise do perfil do paciente como vítima de múltiplos traumas. Cogitare enferm. 2012;17(4):717-23.

9. Conselho Federal de Enfermagem. Resolução n. 358 de 15 de outubro de 2009. Dispõe sobre a Sistematização da Assistência de Enfermagem e a Implementação do Processo de Enfermagem em ambientes, públicos ou privados, em que ocorre o cuidado profissional de Enfermagem e da outras providencias. Brasília; 2009.

10. Risner PB. Diagnosis: analysis and synthesis of data. In: Griffith-Kenney JW, Cristensen PJ. In: Nursing Process: application of theories frameworks, and models. $2^{\mathrm{a}} \mathrm{ed}$. St. Louis: Mosby; 1990. p. 132-57.

11. Flório MCS, Galvão CM. Cirurgia ambulatorial: identificação dos diagnósticos de enfermagem no período perioperatório. Rev. Latino-Am. Enfermagem. 2003;11(5):630-7.

12. Rigotti MA, Ferreira AM. Intervenções de enfermagem ao paciente com dor. Arq. Ciênc. Saúde. [Internet] 2005;12(1) [acesso em 15 jun 2011]. Disponível: http:// www.cienciasdasaude.famerp.br/Vol-12-1/09\%20 -\%20id\%20105.pdf

13. Pitta GBB, Castro AA, Burihan E. Angiologia e cirurgia vascular: guia ilustrado [Internet]. Maceió: UNCISAL/ ECMAL \& LAVA; 2003. [acesso em 17 jun 2011]. Disponível: http://www.lava.med.br/livro

14. Gomes PER, Guerra FCl. Produção Científica de Enfermagem sobre Integridade da Pele Prejudicada: Implicação para a Enfermeira de Cuidados Intensivos [trabalho de conclusão de curso]. Niterói (RJ): Universidade Federal Fluminense/UFF; 2001. [Internet] [acesso em 18 jun 2011]. Disponível: www.uff.br/ nepae/siteantigo/integridadedapele.doc

15. Cavalcante AMRZ, Nakatani AYK, BMM, Garcia TR, Nunes DP, Nunes PS. The analysis of activities not performed by the nursing team regarding the diagnosis of ineffective breathing pattern in the elderly. Rev Esc Enferm USP. [Internet]. 2012;46(3). [acesso em 15 jun 2011]. Disponível: http://dx.doi.org/10.1590/S008062342012000300011 